\title{
Translating Trial Results in Clinical Practice: the Risk GP Model
}

\author{
Jonathan Fuller ${ }^{1}$ • Luis J. Flores ${ }^{2}$
}

Received: 5 March 2016 / Accepted: 25 April 2016/Published online: 4 May 2016

(C) Springer Science+Business Media New York 2016

Keywords Prediction $\cdot$ Knowledge translation $\cdot$ Translational research · Translational medicine - Implementation science . Risk $\cdot$ Generalization $\cdot$ Probability $\cdot$ Clinical trials $\cdot$ Clinical reasoning

$\begin{array}{ll}\text { Abbreviations } \\ \text { AR } & \text { Absolute risk } \\ \text { ARR } & \text { Absolute risk reduction } \\ \text { CVD } & \text { Cardiovascular disease } \\ \text { KT } & \text { Knowledge translation } \\ \text { Risk GP } & \text { Risk generalization-particularization }\end{array}$

The medical research community is fairly unanimous in its agreement that "translating scientific discoveries" and "translating research findings" is important, but achieving translation remains a challenge [1]. There exists a research literature on strategies for knowledge dissemination or transfer [2]. In comparison, an equally important aspect of translation, the incorporation of research findings in clinical reasoning, has been all but ignored. What does a "relative risk reduction of $20 \%$ " in a population of clinical trial participants tell us about whether the drug will work for a particular patient outside the trial?

In this article, we examine the logic of translating therapeutic study results (e.g., clinical trial findings) in clinical practice. The kind of translation we have in mind is often called

Associate Editor Craig Stolen oversaw the review of this article

Jonathan Fuller

jonathan.fuller@mail.utoronto.ca

Faculty of Medicine, University of Toronto, Toronto, Canada

2 Department of Philosophy, King's College London, London, UK knowledge translation (KT) or T2 [1]. Although little explicit attention is given to the logic of translation, we argue that a particular model of translation is implicit in most KT activities; we call it the Risk Generalization-Particularization (Risk GP) Model. Here, we describe the model, including its limitations.

Elsewhere, we argue that the Risk GP Model is the standard model of prediction in prognosis and therapy [3]. In therapy, prediction requires making an inference about a patient's response to treatment. In the Risk GP Model, this involves translating study findings in two stages. In the first stage, generalization, translation involves transportation of the group-level effect size from the trial to a target population. The preferred measures of effect size, particularly in cardiovascular research, are derived from the absolute risk (AR), and include the relative risk (RR) and absolute risk reduction (ARR). For instance, in a meta-analysis of trials, the RR of cardiovascular disease (CVD) events due to a cholesterollowering statin was approximately 0.8 [4]. In the Risk GP Model, the evidence user generalizes this RR of 0.8 to a target population (e.g., patients at high risk of CVD in the USA). In the second stage, particularization, translation involves transformation of the effect size into a change in outcome for a patient in the target population. Thus, we call this model the Risk GP Model.

The inference from populations to individual patients (particularization or "individualization") has received virtually no attention, yet is presupposed whenever clinicians "apply" study results to particular patients. When the effect size is derived from the AR (which quantifies the relative frequency of outcomes/events), the output of particularization is the change in probability of the outcome for the patient. Thus, if a patient in the high risk target population has an untreated CVD risk of $25 \%$, we predict that a statin with a RR of 0.8 will lower their probability of CVD to $20 \%$.

The slip in the meaning of "medical risk" at this stage often goes unnoticed. The RR or ARR measured in the trial is 
derived from the absolute risk, the relative outcome frequency. The clinician particularizes the RR or ARR to determine the change in individual risk [5], or outcome probability for a single patient. It is individual risks or probabilities that are directly relevant to clinical decision-making (e.g., in balancing risk of benefit with risk of harm).

The translation metaphor, though useful, is potentially misleading. Generalization and particularization are not simply a matter of rewriting effect sizes in a different language; they are inferences. Like all models, Risk GP relies upon assumptions. Generalization invokes a "representativeness" or "sufficient similarity" assumption: The trial and target are assumed to be sufficiently similar in the right ways. Widespread worries about the external validity of efficacy trials can be understood as worries that the representativeness assumption fails. Meanwhile, particularization's assumptions include the premise that the patient in question is a randomly presenting member of the target population, an assumption that often hangs on an uncertain diagnosis. A further concern about particularization is that the target populations we define in practice are not particular enough, and thus particularization ignores relevant patient details. Put differently, oftentimes the change in a particular patient's individual risk should not equal the effect size in a heterogeneous population. The personalized and precision medicine movements aim to address this concern in the research phase.

The Risk GP Model and its assumptions (which are frequently problematic) deserve far greater attention. Medicine needs more advanced approaches to translating trial results, which might include Bayesian approaches to modifying risk estimates [3]. More radically, clinicians must sometimes call upon prediction models that do not require translating trial results, such as physiological mechanistic reasoning, $n$-of- 1 studies, and reasoning from clinical experience. We call this strategy of using multiple models "prediction model pluralism" [3].

Researchers and clinicians must pay closer attention to the logic of knowledge translation if they hope to develop a rigorous science of translational medicine. "Closing the research-to-practice gap" requires first understanding how to close the inferential gap between study populations and real-world patients.

Acknowledgments JF gratefully acknowledges funding support from the Canadian Institutes of Health Research. LJF is thankful for funding from the Chilean National Commission for Scientific and Technological Research (CONYCIT).

Compliance with Ethical Standards No human studies and no animal studies were carried out by the authors for this article.

Conflict of Interest The authors declare that they have no conflict of interest.

Sources of Funding Canadian Institutes of Health Research and Chilean National Commission for Scientific and Technological Research (CONYCIT).

\section{References}

1. Woolf, S. H. (2008). The meaning of translational research and why it matters. JAMA, 299(2), 211-213.

2. Fixsen, D. L., Naoom, S. F., Blase, K. A., Friedman, R. M., \& Wallace, F. (2005). Implementation research: a synthesis of the literature. Tampa: National Implementation Research Network, University of South Florida.

3. Fuller, J., \& Flores, L. J. (2015). The risk GP model: the standard model of prediction in medicine. Studies in History and Philosophy of Biological and Biomedical Sciences, 54, 49-61.

4. Baigent, C., Keech, A., Kearney, P. M., Blackwell, L., Buck, G., Pollicino, C., et al. (2005). Efficacy and safety of cholesterollowering treatment: prospective meta-analysis of data from 90,056 participants in 14 randomised trials of statins. Lancet, 366(9493), 1267-1278.

5. Dawid, P. (2015). On individual risk. Synthese doi:10.1007/s11229015-0953-4. 